\title{
453318 - EFFECT OF OUTPATIENT BOWEL PREPARATION ON PREOPERATIVE ELECTROLYTES
}

\author{
Neal Badner, MD, FRCP(C) ${ }^{1}$, Aaron Mocon, B Sc ${ }^{2}$ \\ 1. Anesthesiology, LHSC, London, ON, Canada \\ 2. Faculty of Medicine, University of Western Ontario, London, ON, Canada
}

Introduction: Prior to undergoing an elective bowel resection, patients receive a bowel preparation to clean out the normal bacterial flora. This bowel preparation allows surgeons to perform a primary anastomosis. In the past, this was performed on an inpatient basis with concomittant intravenous rehydration. With the shift to outpatient surgery, the bowel preparation is now also performed on an outpatient basis. This has the potential to cause electrolyte abnormalities and for this reason, patients have their electrolytes repeated on the day of surgery in order to detect and rectify any abnormalities. Rarely, however are any abnormalities detected and though the ability to do this on an outpatient basis has been studied, to our knowledge the effect on preoperative bloodwork has not been documented. If changes are minimal or nonexistant, patients are needlessly receiving phlebotomies and the medical system is wasting money on unnecessary laboratory testing.

Methods: Following REB approval, charts of patients 18 years of age and older, who underwent outpatient bowel preparation for an elective bowel resection in 2006 at a tertiary care institution who had had both preadmission and day of surgery bloodwork obtained (standard practise) were reviewed. Patients having emergency surgery or were inpatients prior to the procedure were excluded. Demographic data, coexisting medications and diseases, the type, timing and amount of bowel preparation as well as preadmission clinic and then day of surgery bloodwork were recorded. Statistical analysis included paired t-tests for demogrphic data and chi-squared analysis for nonparametric data. Multiple logistic regression was used to determine if there were any predictive factors for patients who developed hypokalemia or elevated creatinine.

Results: There were no differences in demographic data. The table compares the preadmision clinic and day of surgery bloodwork data. There was a statistically significant decrease in potassium and BUN and an increase of creatinine as shown. No correlation between demographic factors and day of OR hypokalemia or elevated creatinine were noted.

Discussion: We documented statistically significant but clinically insignificant changes in potassium, BUN and creatinine. There were no predictive factors for hypokalemia and elevated creatinine. This questions the need for repeating electrolytes on the day of surgery when having an outpatient bowel preparation.

References: 1. Ezri T et al. CJA 2006;53:153-8. 2. Holte K et al. Dis Colon Rectum 2004;47:1397-1402.

Comparison of Preadmission Clinic (PAC) \& Day of OR Bloodwork 


\begin{tabular}{|c|c|c|c|}
\hline & PAC & Day OR & p value \\
\hline $\mathrm{Na}$ & $138.9+/-2.0$ & $139.0+/-2.5$ & 0.68 \\
\hline $\mathrm{Cl}$ & $101.9+/-3.4$ & $102.3+/-3.8$ & 0.34 \\
\hline $\mathrm{HCO} 3$ & $28.0+/-2.1$ & $27.6+/-2.2$ & 0.27 \\
\hline $\mathrm{K}$ & $4.0+/-0.4$ & $3.7+/-0.5$ & $<.001$ \\
\hline $\mathrm{BUN}$ & $4.8+/-2.3$ & $1.1+/-1.7$ & $<.001$ \\
\hline $\mathrm{Cr}$ & $86+/-25$ & $91+/-26$ & 0.001 \\
\hline $\mathrm{Hgb}$ & $124+/-26$ & $110+/-19$ & \\
\hline
\end{tabular}

